\title{
Conjunctival UV Autofluorescence - Prevalence and Risk Factors
}

James S Wolffsohn BSc (Hons) PhD PgCertHE PgDipAdvClinOptom MBA MCOptom FHEA FBCLA FAAO

Tom Drew BEng PhD AMIMechE

Anna Sulley BSc (Hons) MCOptom FBCLA FAAO *

Ophthalmic Research Group, Aston University, Birmingham, UK $\quad$ * Johnson \& Johnson Vision Care, Wokingham, Berkshire, UK

\begin{abstract}
Purpose: Autofluorescence of ultraviolet (UV) light has been shown to occur in localised areas of the bulbar conjunctiva, which map to active cellular changes due to UV and environmental exposure. This study examined the presence of conjunctival UV autofluorescence in eye care practitioners (ECPS) across Europe and the Middle East and its associated risk factors.
\end{abstract}

Method: Images were captured of 307 ECPs right eyes in the Czech Republic, Germany, Greece, Kuwait, Netherlands, Sweden, Switzerland, United Arab Emirates and the United Kingdom using a Nikon D100 camera and dual flash units through UV filters. UV autofluorescence was outlined using ImageJ software and the nasal and temporal area quantified. Subjects were required to complete a questionnaire on their demographics and lifestyle including general exposure to UV and refractive correction.

Results: Average age of the subjects was $38.5 \pm 12.2$ years (range 19-68) and $39.7 \%$ were male. Sixty-two percent of eyes had some conjunctival damage as indicated by UV autofluorescence. The average area of damage was higher $(p=0.005)$ nasally $(2.95 \pm 4.52$ $\left.\mathrm{mm}^{2}\right)$ than temporally $\left(2.19 \pm 4.17 \mathrm{~mm}^{2}\right)$. The area of UV damage was not related to age $(r=$ $0.03, p=0.674)$, gender $(p=0.194)$, self-reported sun exposure lifestyle $(p>0.05)$, geographical location $(p=0174)$, sunglasses use $(p>0.05)$ or UV-blocking contact lens use $(p>0.05)$, although it was higher in those wearing contact lenses with minimal UV-blocking and no spectacles $(p=0.015)$. The area of UV damage was also less nasally in those who wore contact lenses and spectacles compared to those with no refractive correction use $(p=$ 0.011 nasal; $p=0.958$ temporal).

Conclusion: UV conjunctival damage is common even in Europe, Kuwait and UAE, and among ECPs. The area of damage appears to be linked with the use of refractive correction, with greater damage nasally than temporally which may be explained by the peripheral light focusing effect.

Sponsor: Johnson \& Johnson Vision Care (Johnson \& Johnson Vision Care is a part of Johnson \& Johnson Medical Ltd) 


\section{$\underline{\text { Introduction }}$}

Cells have molecules that fluoresce when they are excited by ultraviolet (UV) radiation of appropriate wavelength. When the light emission occurs from stimulation of endogenous cellular components, it is termed autofluorescence. Most cellular autofluorescence is derived from lysosomes and mitochondria. ${ }^{1}$ Autofluorescence of UV light has been shown to occur in localised areas of the bulbar conjunctiva. It is detected in children and adults, highlighting visible pingueculae, but also in some subjects without any visible slit-lamp conjunctival changes. ${ }^{2,3}$ The location of the UV autofluorescence appears to map to active cellular changes within the conjunctiva, in areas known to be susceptible to UV and environmental exposure to wind and dust damage, resulting in pterygia and pingueculae. ${ }^{4}$

A recent study was conducted of around 640 people from Norfolk Island, Australia, which is geographically isolated, has a stable population with restricted migration, along with consistent sun/UV exposure and low levels of pollution. ${ }^{5-7}$ The area of autofluorescence declined with age, covered a larger area in males, but there was no statistical difference between eyes or with systemic co-morbidity. ${ }^{5}$ Autofluorescence was greater nasally than temporally, which may be explained by the peripheral light focusing effect, with only about $3 \%$ of the population showing no detectable autofluorescence. The peripheral light focusing effect is the intensification of the light intensity incident on the temporal corneal optics across to the temporal limbal and crystalline lens regions. ${ }^{8}$ It has also been linked with pterygia, ${ }^{3}$ and is reported to be related to myopia ${ }^{6}$ although this may be due to the association of both conditions with time spent outdoors. ${ }^{7}$ Autofluorescence has been shown to 
occur in children from about the age of nine, suggesting monitoring and education in prevention strategies would be worthwhile. $^{2}$

The studies to date have been conducted largely on an island off Australia, where the level of exposure to UV damage is likely to be higher due to an outdoor lifestyle, subtropical climate and damage to the ozone layer. Hence this study examined whether the effects occurred across a more diverse population in the Northern hemisphere and equatorial region. 


\section{Methodology}

UV autofluorescence images were captured of the eyes of 307 eye care practitioners (ECPs) in the Czech Republic, Germany, Greece, Kuwait, Netherlands, Sweden, Switzerland, United Arab Emirates and the United Kingdom, following informed consent, as part of educational events conducted by Johnson \& Johnson Vision Care in late 2012 and early 2013. The study was approved by the Aston University Ethics Committee (Ref 545 2013) and conformed to the tenet's of the Declaration of Helsinki.

Conjunctival UV autofluorescence photographs were taken using the camera system developed by Coroneo and colleagues. ${ }^{2,4}$ This consisted of a Nikon D100 (Nikon, Melville, New York, NY, USA) digital camera and 105 mm f/2.8 Micro Nikkor (Nikon, Melville) lens fitted with infra-red and UV barrier filters (B\&W 489 and B\&W420 and rotating polariser; transmittance range $300-400 \mathrm{~nm}$, peak $365 \mathrm{~nm}$ ) as an excitation source, so primarily the UV autofluorescence was captured by the camera sensor . The flash unit was a Metz 36C-2 (Zirndorf, Germany - guide number $36(\mathrm{~m})$ / ISO $100 / 21^{\circ}$ ) overlaid with Wratten 2E and 18A UV (Kodak, Rochester, New York, USA) transmission filters. Images were saved in RGB format at the D100 JPEG Fine (1:4 compression) and high resolution settings. Nasal and temporal images were captured at $0.94 \mathrm{X}$ magnification, with the eyes viewing a fixation target approximately $35^{\circ}$ from the camera optical axis. Due to the association between right and left eyes, only data from right eyes was included in the analysis to avoid statistical bias. Quantitative analysis of the UV autofluorescence images was conducted using ImageJ software (http://rsbweb.nih.gov/ij/) to subjectively outline the edges of the conjunctival area fluorescing, and calculating the area in pixels by a 
masked researcher. This was converted to square millimetres by calibrating the pixels to millimetres from an image of a ruler using the same camera system (Figure 1). Intra-observer and inter-observer repeatability using similar methodology has been shown to be good. ${ }^{7}$

Figure 1: Image analysis of the area of UV autofluorescence using ImageJ

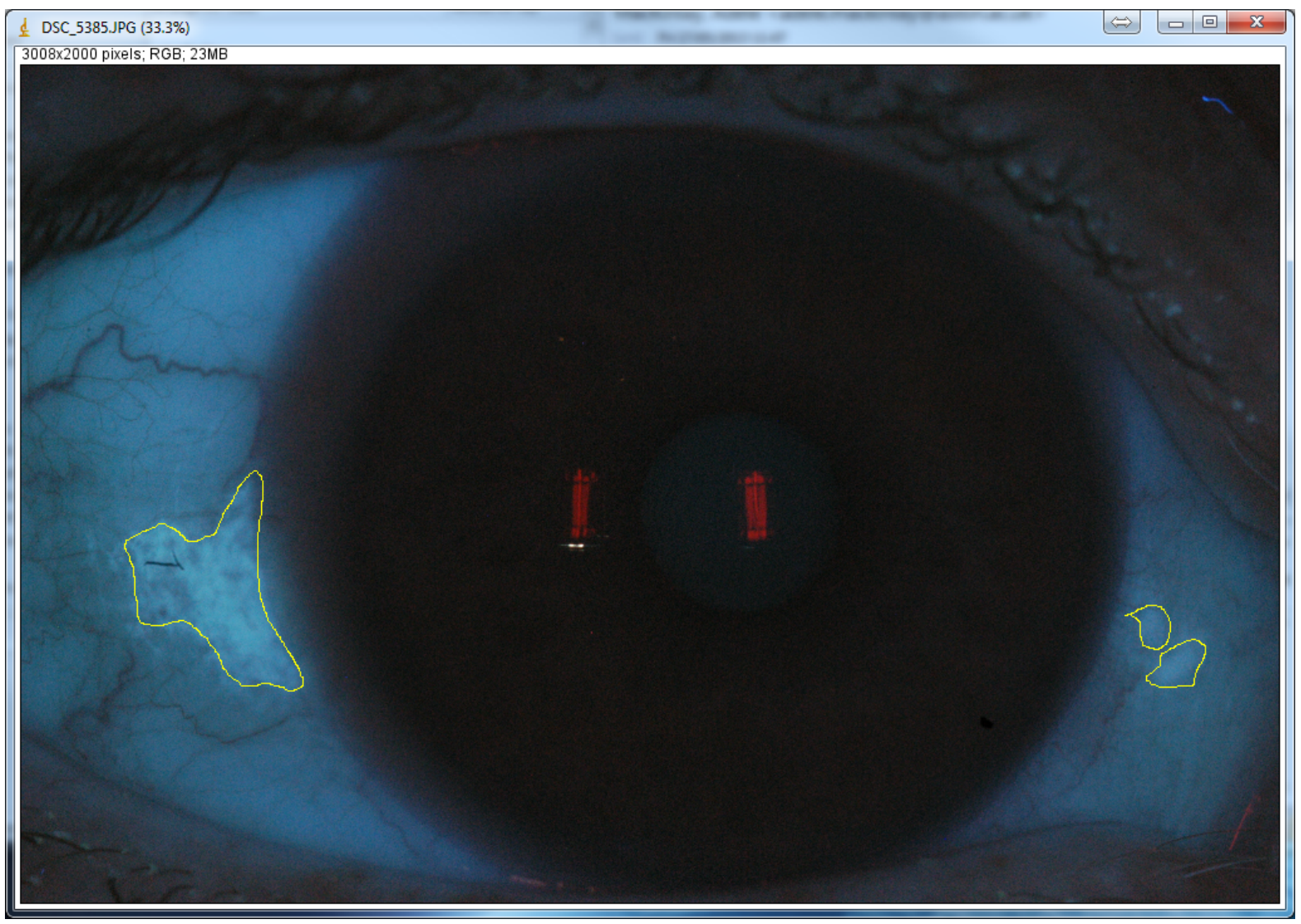

Subjects were required to complete a short questionnaire relating to their demographics (age and gender), refractive correction (spectacles and contact lenses, if worn), lifestyle (self-reported as 'sun-avoider', 'average sun exposure' or 'sun-worshipper', usual habitat - northern or southern hemisphere or equatorial region, and use of sunglasses - worn most of time outdoors, worn only when sunny, worn sometimes or never worn) and contact lens details (number of years lenses worn, current brand and years worn, contact lens history). 


\section{Statistics}

As the area of UV autofluorescence (in $\mathrm{mm}^{2}$ ) was not normally distributed (onesample Kolmogorov-Smirnov test $p<0.001)$, non-parametric statistics were used. Poor images where no image of the background eye was perceptible were excluded from the analysis, but as these were caused by operator error and occurred across data collection sites, no bias was evident. 
$\underline{\text { Results }}$

Subjects examined in the study had a mean age of 38.5 years $( \pm 12.2$ years, range 19-68 years) and 39.7\% were male. Twenty-six (8.5\%) reported being from the equatorial region, two from the southern hemisphere, 219 from the northern hemisphere (71.3\%) and there were 60 non-responders (19.5\%). Sixty-six $(21.5 \%)$ reported being a sun-worshipper, 51 (16.6\%) a sun-avoider, with 162 (52.8\%) reporting average sun exposure with no data on 28 . Seventy-nine $(25.7 \%)$ wore no refractive correction, 35 (11.4\%) contact lenses only, $82(26.7 \%)$ spectacles only and $82(26.7 \%)$ a combination of both, with 29 (9.4\%) with no data. Twelve (3.9\%) never wore sunglasses, 67 (21.8\%) wore sunglasses most of the time, 94 (30.6\%) only when sunny, $74(24.1 \%)$ sometimes and there were no data on 60 (19.5\%) subjects. Finally, of the 307 subjects, 45 (14.7\%) wore UV-blocking contact lenses, $38(12.4 \%)$ wore contact lenses with minimal UV blocking and 18 (5.9\%) had worn a mix of both.

Sixty-two percent of eyes had some conjunctival damage as indicated by UV autofluorescence (Figure 2). The average area of damage was higher (related sample Wilcoxon Signed Rank Test $p=0.005)$ nasally $\left(2.95 \pm 4.52 \mathrm{~mm}^{2}\right)$ than temporally $\left(2.19 \pm 4.17 \mathrm{~mm}^{2}\right)$. 


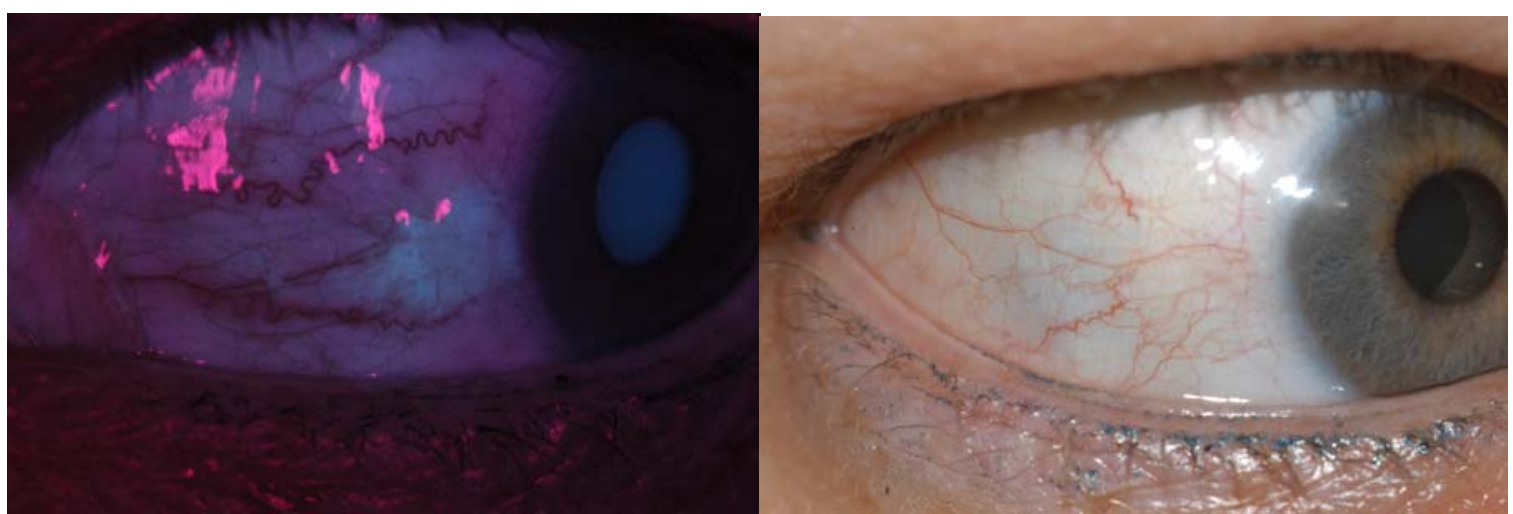

Figure 2: $\quad$ Example images of eye taken with Coroneo camera $(A)$ and with standard white light (B).

The amount of UV damage (average area quoted where no difference between nasal and temporal regions) was not related to age $(r=0.03, p=0.674$; Figure 3$)$, gender (male $2.69 \pm 4.19 \mathrm{~mm}^{2}$; female $2.27 \pm 3.33 \mathrm{~mm}^{2}$; independent sample MannWhitney $U$ test $p=0.194$ ), reported sun exposure lifestyle (independent sample Mann-Whitney $U$ test $p=0.358$ nasal, $p=0.777$ temporal; Figure 4), geographical location (northern hemisphere $2.62 \pm 3.67 \mathrm{~mm}^{2}$; equatorial $3.42 \pm 5.60 \mathrm{~mm}^{2}$; independent sample Mann-Whitney $U$ test $p=0.174$ ), sunglasses use (independent sample Mann-Whitney $U$ test $p=0.112$ nasal, $p=0.639$ temporal; Figure 5), or UVblocking contact lenses compared to those who wore contact lenses with minimal UV blocking (nasal: $2.14 \pm 3.51 \mathrm{~mm}^{2}$ vs $3.66 \pm 5.96 \mathrm{~mm}^{2}$ respectively; independent sample Mann-Whitney $U$ test $p=0.774$; temporal: $1.77 \pm 2.96 \mathrm{~mm}^{2}$ vs $1.94 \pm$ $3.59 \mathrm{~mm}^{2}$ respectively; independent sample Mann-Whitney $U$ test $p=0.723$ ). For those wearing UV blocking contact lenses, there was no difference in autofluorescene area between those who used them as their primary visual correction $\left(2.36 \pm 2.16 \mathrm{~mm}^{2}\right)$ and those that who wore spectacles as well $(1.95 \pm$ $3.00 \mathrm{~mm}^{2}$; independent sample Mann-Whitney $U$ test $\mathrm{p}=0.687$ ). However, for those wearing contact lenses with minimal UV blocking, there was a larger autofluorescene 
area in those who used them as their primary visual correction $\left(5.50 \pm 6.67 \mathrm{~mm}^{2}\right)$ and those that who wore spectacles as well $\left(1.90 \pm 2.79 \mathrm{~mm}^{2}\right.$; independent sample Mann-Whitney $U$ test $p=0.015$ ).

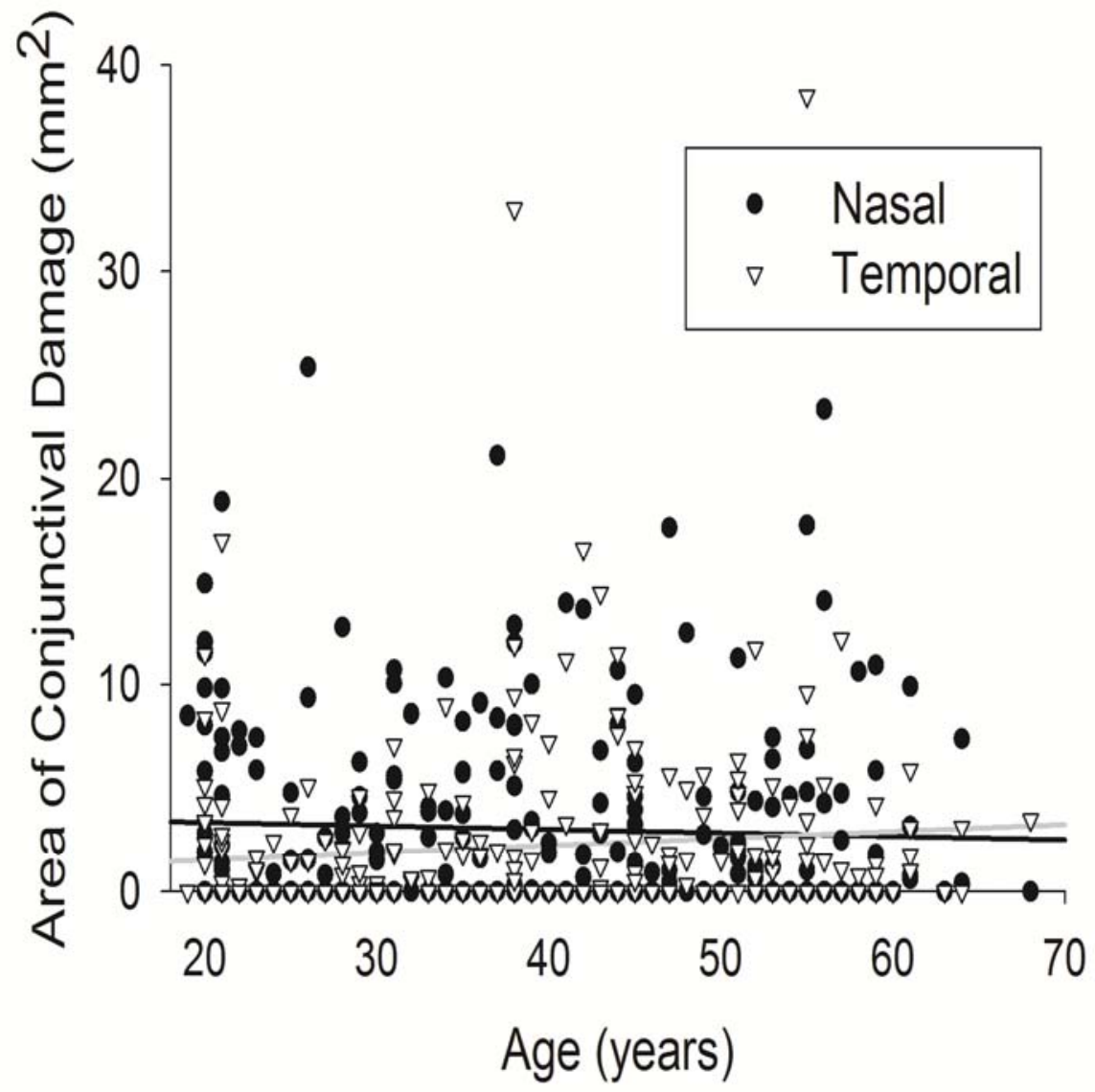

Figure 3: UV autofluorescence area with age on the nasal (black) and temporal (white) conjunctiva. $\mathrm{N}=307$. 


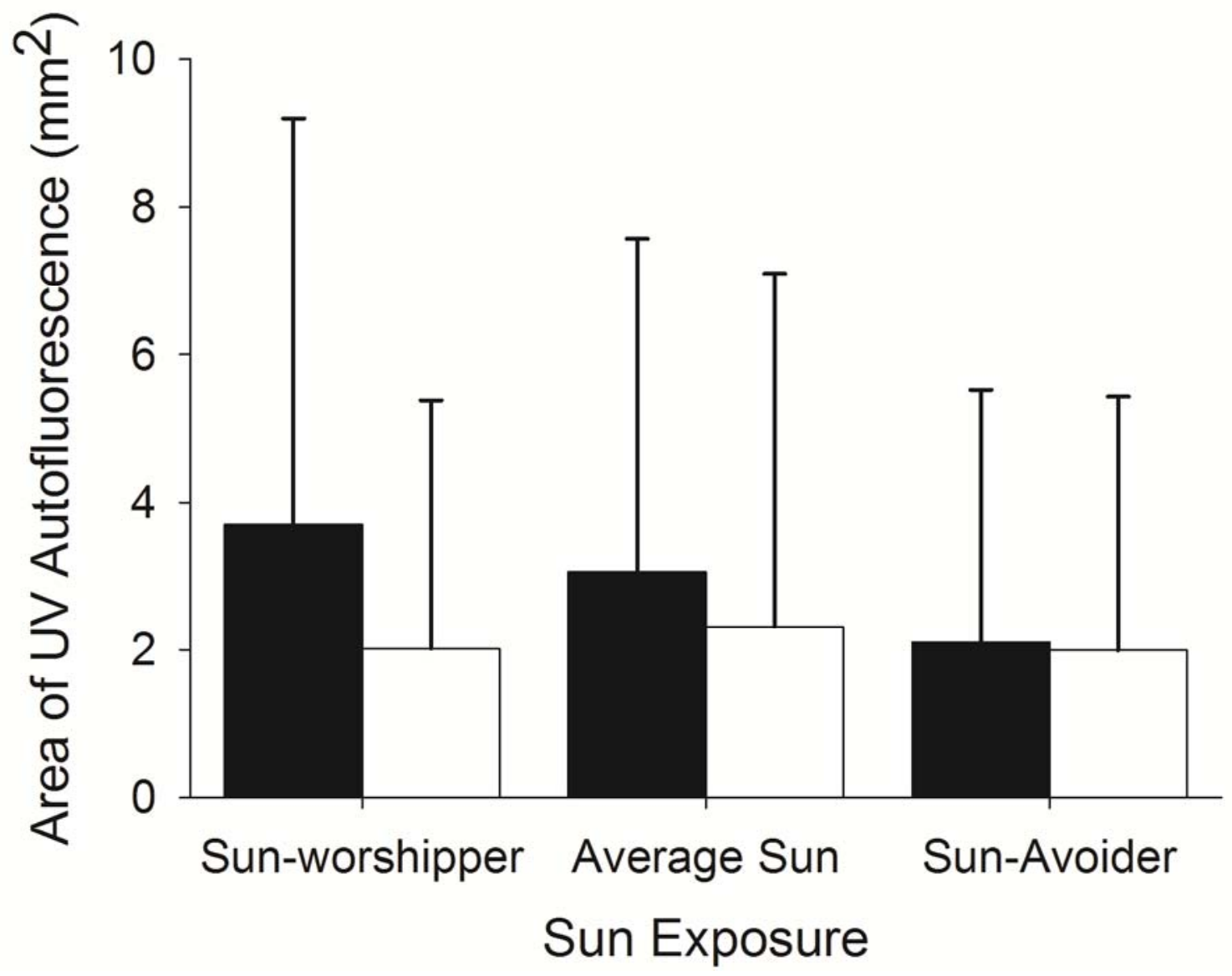

Figure 4: $\quad$ UV autofluorescence area with sun exposure on the nasal (black) and temporal (white) conjunctiva. $\mathrm{N}=280$. 


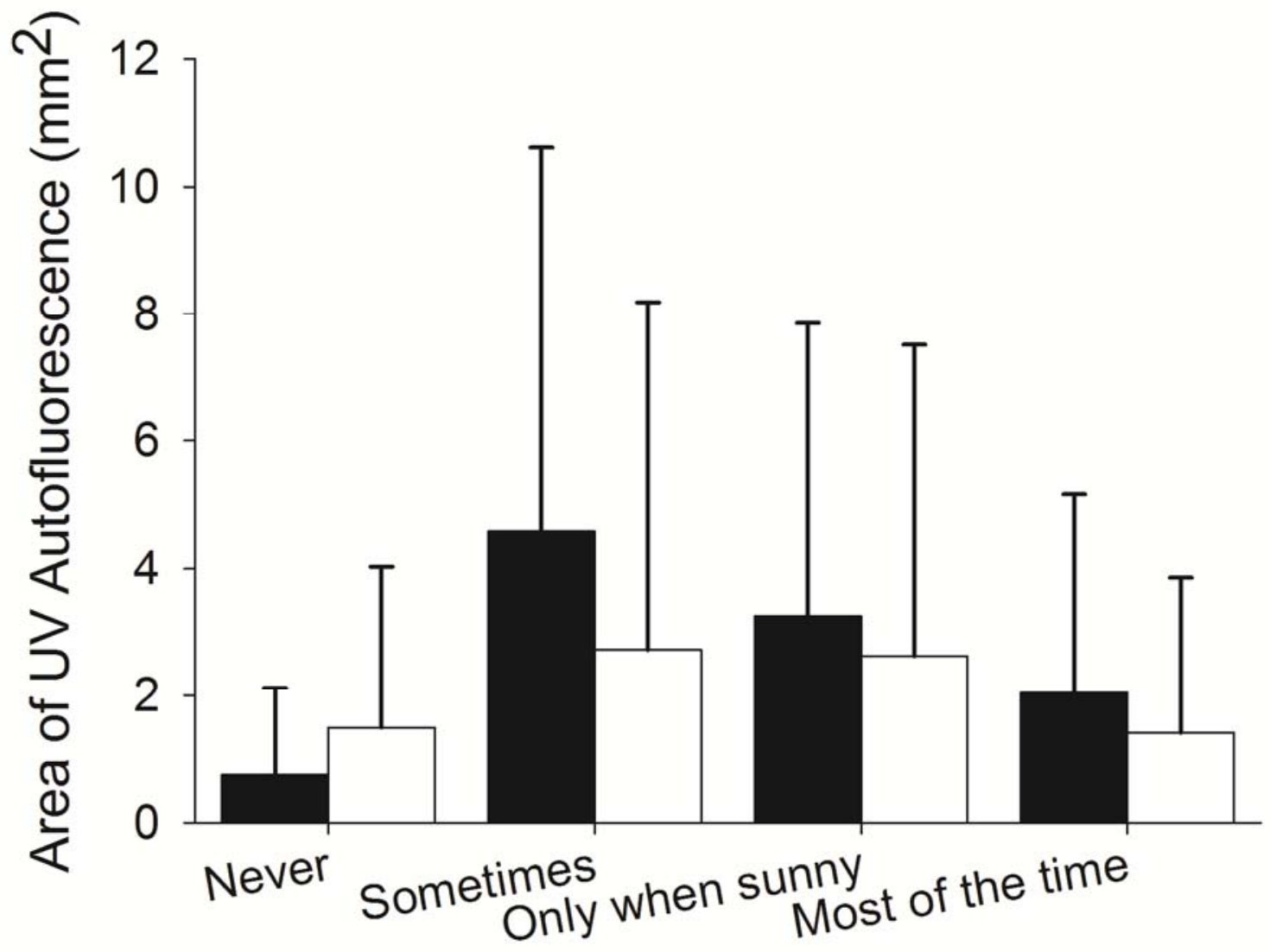

\section{Sunglasses Wear}

Figure 5: $\quad$ UV autofluorescence area with sunglasses usage on the nasal (black) and temporal (white) conjunctiva. $\mathrm{N}=248$.

However, UV damage was greater nasally in those who wore no refractive correction compared to those who wore both contact lenses and spectacles (independent sample Mann-Whitney $U$ test $p=0.011$ nasal, $p=0.958$ temporal; Figure 6$)$. 


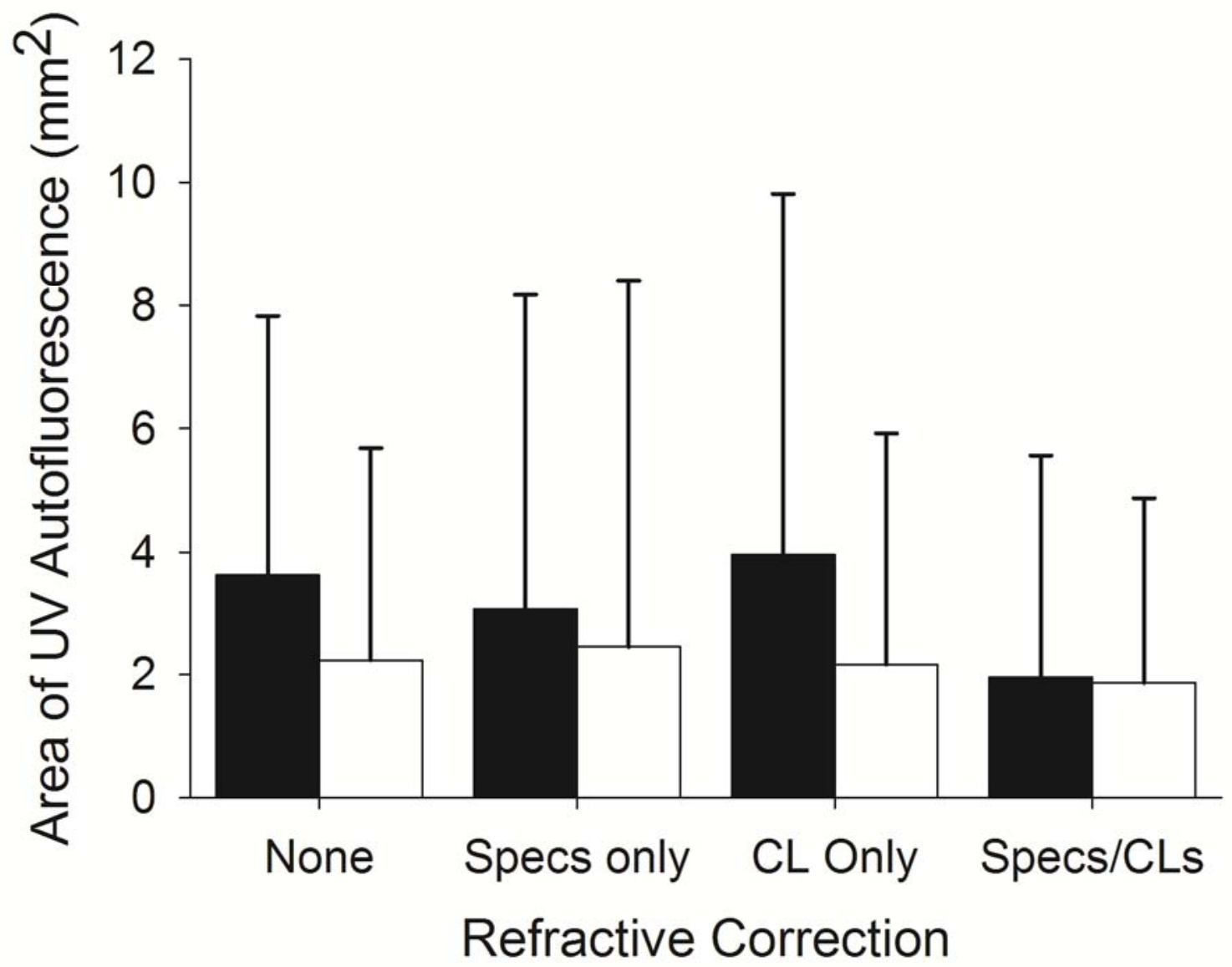

Figure 6: UV autofluorescence area with refractive correction on the nasal (black) and temporal (white) conjunctiva. $\mathrm{N}=280$. 


\section{Discussion}

This study examined whether UV autofluorescence identified damage ${ }^{4}$ to the conjunctiva occurred across a diverse population in the Northern hemisphere and equatorial region. Although the percentage of the population studied with UV autofluorescence (62\% was less than in the sub-tropical Norfolk Island study (96\%), ${ }^{5}$ still nearly two thirds of the subjects showed some UV autofluorescence, indicating the damage is more widespread than might be expected based on the largely northern hemisphere cohort (89\%). As with the findings of Sherwin and colleagues, ${ }^{5}$ the damage was greater nasally than temporally, presumed to be due to the peripheral light focusing effect, where rays of light tangential to the anterior eye, which can bypass most non-wraparound sunglasses, are intensified by the optics of the eye, focusing on the nasal limbal corneal and crystalline lens regions. ${ }^{8}$

However, in the cohort examined in this study, the area of autofluorescence did not decline with age nor did the damage cover a larger area in males, as found by Sherwin and colleagues. ${ }^{5}$ This is unlikely to be due to the difference in sample size as the lack of effect was not close to significance. The lack of effect may be attributed to the less intense sunlight experienced by the population in Europe as, in general, the area of damage was small in this study $\left(2.58 \pm 3.73 \mathrm{~mm}^{2}\right)$ compared to that reported in Norfolk Islanders $\left(17.5 \mathrm{~mm}^{2}\right.$; range 0 to $\left.114 \mathrm{~mm}^{2}\right) .{ }^{5}$ However, the amount of UV damage was also not related to reported sun exposure, although the results showed a higher effect nasally and less damage area with less reported exposure as expected. The subjectivity of reporting sun exposure is of course great and will limit the ability to observe a significant result. Roughly half of the subjects reported having average sun exposure, with close to a quarter stating they were a 
sun-worshipper and a quarter stating they were a sun-avoider. In addition, damage appeared to be higher in the cohort living in an equatorial region (Dubai, United Arab Emirates), but not statistically significantly so. It would be expected that UV damage would increase with age, if at all, due to chronic exposure lifestyle, which is opposite to the finding of the Australian study.

Sunglasses use again had no significant effect on detected UV autofluorescenceindicated damage, as previously reported by Sherwin and colleagues7 with sunglasses or hat use, although interestingly the similarity was greater on the temporal side, suggesting if anything a difference in the nasal region. As has been reported in survey data, many subjects (about one third) only wear sunglasses in sunny conditions and a further quarter only sometimes, indicating the need for better education regarding the use of protection from the transmission of UV into the eye since UV can pass through clouds.

There was no statistical difference between the UV autofluorescence-indicated damage in those wearing UV-blocking contact lenses compared to contact lenses with minimal UV blocking, but only about a quarter of the cohort (27\%) wore contact lenses (evenly split between UV and minimal UV blocking) and the difference between these lens types was larger nasally (on average by $1.4 \mathrm{~mm}^{2}$ ) than temporally (on average by $0.17 \mathrm{~mm}^{2}$ ) as would be expected due to the protection offered by UV-blocking contact lenses from transmission of UV light that enters the eye peripherally. Those subjects who wore soft contact lenses with minimal UV blocking as their only refractive correction showed more UV autofluorescenceindicated damage than those who wore spectacles as well, whereas those that wore 
UV blocking contact lenses did not show this difference. CR39 has a UVB blocking transmission, which despite allowing peripheral light to reach the ocular surface seems to prevent UV conjunctival damage, whereas UV blocking contact lenses offer similar protection even if spectacles are not worn.

Interestingly, UV damage was greater nasally in those who wore no refractive correction compared to those who used both contact lenses and spectacles, regardless of the UV-blocking properties of the lenses and there was no difference in those who principally wore contact lenses, leaving this finding unexplained. Similar proportions of subjects, roughly a quarter, wore no refractive correction, spectacles only or a combination of spectacles and contact lenses, with just under half this proportion wearing contact lenses only. This is higher than the uptake rate of contact lenses across Europe, but is most likely explained by the subjects being ECPs with easier and less costly access to contact lenses. The proportion of subjects who reported wearing UV-blocking contact lenses compared to contact lenses with minimal UV blocking (14.7\% vs $12.4 \%$ respectively, with $5.9 \%$ having worn a mixture of both) suggests this informed group may have influenced the proportion wearing UV protection compared to currently available contact lenses.

Subjects examined in the study reasonably reflected the general population being, on average, middle aged and roughly equal gender split. It was presumed that, as ECPs, the subjects in the study would have a relatively reliable recollection of their refractive correction compared to that of the general population. The study was limited by the quality of the images (approximately 200 subjects were rejected as having images that could not be graded), missing data in those included in the 
questionnaire (about 5\%) and the quality of self-reported lifestyle and refractive correction data. Future studies could be directed at assessing conjunctival UV autofluorescence in a larger sample population, with a real-time analysis system, among a wider age range and at different geographical locations.

In conclusion, the peripheral light focusing effect is appears to be have a role in UV auto fluorescence-indicated damage, and a large proportion of adults show some UV conjunctival damage even in climates with less intense sun exposure. This may be in part due to the lower solar angle avoiding the eyes natural brow and eyelid protection.9 Hence ECPs should communicate the potential for damage and the potential forms of UV protection to all patients. For the future, there may be a public health need to incorporate the imaging of UV autofluorescence into routine clinical practice to educate patients and advise on ways to protect eyes from the transmission of UV radiation.

\section{Acknowledgement}

Study supported by Johnson \& Johnson Vision Care, part of Johnson \& Johnson Medical Ltd.

Thanks to eye care practitioners and THE VISION CARE INSTITUTE ${ }^{\circledR}$ for the data from the educational events.

Thanks to Professor Minas Coroneo for the use of his camera. 


\section{References}

1. Monici M. Cell and tissue autofluorescence research and diagnostic applications. Biotechnol Annu Rev 2005;11:227-56.

2. Ooi J-L, Sharma NS, Papalkar D, Sharma S, Oakey M, Dawes P, Coroneo MT. Ultraviolet fluorescence photography to detect early sun damage in the eyes of school-aged children. Am J Ophthalmol 2006;141:294-8.

3. Sherwin JC, Hewitt AW, Kearns LS, Griffiths LR, Mackey DA, Coroneo MT. The association between pterygium and conjunctival ultraviolet autofluorescence: The Norfolk Island Eye Study. Acta Ophthalmologica 2013;91:363-70.

4. Ooi J-L, Sharma NS, Sharma S, Papalkar D, Oakey M Dawes P, Coroneo MT. Ultraviolet fluorescence photography: Patterns in established pterygia. Am J Ophthalmol 2007;143:97-101.

5. Sherwin JC, Hewitt AW, Kearns LS, Coroneo MT, Griffiths LR, Mackey DA. Distribution of conjunctival ultraviolet autoflourescence in a population-based study: the Norfolk Island Eye Study. Eye 2011a;25:892-9.

6. Sherwin JC, Hewitt AW, Kearns LS, Coroneo MT, Kearns LS, Griffiths LR, Mackey DA. The association between time spent outdoors and myopia using a novel biomarker of outdoor light exposure. Invest Ophthalmol Vis Sci 2012b;53:4363-70.

7. Sherwin JC, McKnight CM, Hewitt AW, Griffiths LR, Coroneo MT, Mackey DA. Reliability and validity of conjunctival ultraviolet autofluorescence measurement. $\mathrm{Br} \mathrm{J}$ Ophthalmol 2012a;96:8015.

8. Coroneo MT, Mullerstolzenburg NW, Ho A. Peripheral light focusing by the anterior eye and the ophthalmohelioses. Ophthalmic Surg lasers 1991:22;705-11.

9. Hu LW, Gao Q, Gao N, Liu GC, Wang Y, Gong HZ, Liu Y. Solar UV exposure at eye is different from environmental UV: Diurnal monitoring at different rotation angles using a manikin. J Occupational Environ Hygiene 2013;10:17-25. 


\section{Highlights for Review}

- Autofluorescence of ultraviolet (UV) light occurs in localised areas of the bulbar conjunctiva, which map to active cellular changes.

- The study examined conjunctival UV autofluorescence in eye care practitioners across Europe and the Middle East and its associated risk factors.

- UV damage area was not related to age, gender, self-reported sun exposure lifestyle, geographical location, sunglasses use or UV-blocking contact lens use

- It was higher in those wearing contact lenses with minimal UV-blocking and no spectacles.

- The area of UV damage was also less nasally in those who wore contact lenses and spectacles compared to those with no refractive correction use. 